\title{
Cribriform adenocarcinoma of the lung: clinicopathologic, immunohistochemical, and molecular analysis of 15 cases of a distinctive morphologic subtype of lung adenocarcinoma
}

\author{
Alexander C Mackinnon Jr, Arturo Luevano ${ }^{1}$, Lisley C de Araujo ${ }^{2}$, Nagarjun Rao, \\ Min Le and Saul Suster \\ Department of Pathology, Medical College of Wisconsin, Milwaukee, WI, USA
}

\begin{abstract}
Lung adenocarcinoma is characterized by marked heterogeneity and may be composed of an admixture of histologic growth patterns, including acinar, papillary, solid, and lepidic (bronchioloalveolar). Tumors displaying a prominent or predominant cribriform architecture are rare and most often confused for metastases from other organs. We report the clinical, histologic, immunohistochemical, and molecular features in 15 primary lung adenocarcinomas with a predominant cribriform histology. All patients were adults between 30 and 80 years of age (median: 64), and all but one reported a history of heavy cigarette smoking. All cases showed a predominant $(>70 \%)$ cribriform architecture that resembled a variety of tumors arising in other organs, including breast, prostate, ovary, pancreas, uterus, colon, and thyroid. Immunohistochemical stains showed a phenotype consistent with a primary lung tumor (ie, TTF1 + /CK $7+$ ), with negative results for other markers. Molecular analysis in six cases showed that none harbored an EGFR-activating mutation. KRAS mutation was detected in one case, and an ALK1 and ROS1 gene rearrangement were each detected in an additional two cases. Cribriform adenocarcinomas of the lung represent a distinctive histologic subtype of lung cancer that may be morphologically difficult to differentiate from metastases with a predominant cribriform architecture.
\end{abstract}

Modern Pathology (2014) 27, 1063-1072; doi:10.1038/modpathol.2013.227; published online 3 January 2014

Keywords: ALK1; cribriform adenocarcinoma; lung adenocarcinoma; KRAS; metastases to the lungs; molecular diagnostics; ROS1

Non-small-cell lung carcinoma, particularly adenocarcinoma, remains the leading cause of cancer mortality in the United States. ${ }^{1}$ According to the current World Health Organization classification of lung adenocarcinoma, the most frequent type is mixed adenocarcinoma, an invasive tumor with a combination of different histologic subtypes (bronchioalveolar, acinar, papillary, and solid with

Correspondence: Dr S Suster, MD, Department of Pathology, Medical College of Wisconsin, 9200 W Wisconsin Avenue, Milwaukee, WI 53226, USA.

E-mail: ssuster@mcw.edu

This work was carried out while these authors served as International Visiting Fellows in Pathology at the Medical College of Wisconsin.

${ }^{1}$ Current address: Dr Luevano is currently at the ChristusMugerza del Parque Hospital, Chihuahua, México.

${ }^{2}$ Current address: Dr Araujo is currently at Brasilia University Hospital, Brasilia, Brazil.

Received 11 July 2013; revised 29 October 2013; accepted 30 October 2013; published online 3 January 2014 mucin). ${ }^{2,3}$ Although the classification is widely applied, the subtyping has high interobserver variability $^{4,5}$ and lacks a reliable correlation with molecular characteristics. ${ }^{6}$ The international multidisciplinary classification of lung adenocarcinoma recently published by the International Association for the Study of Lung Cancer/American Thoracic Society/European Respiratory Society proposes to classify these tumors according to the predominant histologic component in order to improve molecular and prognostic correlations. ${ }^{7}$

The acinar pattern of lung adenocarcinoma is defined as a neoplasm composed of small round glands, and according to the new International Association for the Study of Lung Cancer/American Thoracic Society/European Respiratory Society classification, it is the predominant component in $30-40 \%$ of cases. ${ }^{6,8}$ A cribriform pattern of growth is not currently acknowledged in the histologic classification of lung adenocarcinoma, although some authors have regarded tumors with a cribriform 
morphology as a variant of the acinar pattern. ${ }^{9}$ In primary lung tumors, a pure or predominant cribriform architecture is rare; most tumors displaying this type of architecture have been commonly regarded as metastases, particularly from the prostate, breast, and colon. ${ }^{3,10,11}$

Mutations in the epidermal growth factor receptor (EGFR) and the KRAS genes, and rearrangements in the anaplastic lymphoma receptor tyrosine kinase $(A L K)$, the c-ros oncogene 1, receptor tyrosine kinase (ROS1) genes, and the RET proto-oncogene are among the most frequently observed molecular alterations in lung adenocarcinomas. ${ }^{4,12} A L K, R O S 1$, and RET gene rearrangements occur exclusively of EGFR or KRAS mutations with rare exception.

The presence of activating mutations of the EGFR gene correlates with a better response to treatments with tyrosine kinase inhibitors (TKIs). ${ }^{13-16}$ The most frequent type of $A L K$ rearrangement is a fusion with the echinoderm microtubule-associated protein-like 4 (EML4). ROS1 gene rearrangements were first reported in $2007^{17}$ and are present in $1-2 \%$ of lung adenocarcinoma. Similar to $A L K$-positive lung cancer, ROS1 gene rearrangements are more common in young, female, never smokers, and tumors harboring these rearrangements demonstrate more favorable response to crizotinib. RET gene rearrangements were recently described, ${ }^{18-20}$ occur in $1-2 \%$ of lung adenocarcinoma, and may also show response to RET-targeted therapy. ${ }^{21,22}$

Recently, testing guidelines for properly selecting lung cancer patients for EGFR and ALK TKIs have been published. ${ }^{23}$ The major recommendation to guide selection of appropriate targeted therapy is testing of all patients with advanced stage lung adenocarcinoma for activating EGFR mutations and $A L K$ gene rearrangements regardless of clinical risk factors or tumor histology. KRAS mutations are present in $15-30 \%$ of lung adenocarcinomas and are regarded as a marker of resistance to TKI treatment. There are no recommendations for routine KRAS, ROS1, and RET testing in lung cancer. ${ }^{7,16,24}$

We report the clinical, histologic, and immunohistochemical status in 15 primary lung adenocarcinomas with a predominant cribriform histologic growth pattern, with special emphasis on the histopathologic differential diagnosis. We also describe the EGFR and KRAS mutational status and the $A L K, R O S 1$, and RET rearrangements in six of these cases for which material was available for analysis, and we discuss the role of molecular testing in this group of tumors.

\section{Materials and methods}

\section{Cases}

A total of 15 cases were identified during a retrospective review of lung adenocarcinomas from the surgical pathology files of the Medical College of
Wisconsin, Milwaukee, Wisconsin (six cases), and the Ohio State University, Columbus, Ohio (nine cases), during a period from 1990 to 2010 . The study was conducted with Institutional Review Board approval from both institutions. Clinical information and follow-up data were obtained from the patients' records or by contacting the responsible physicians. Criteria for inclusion were the presence of $>70 \%$ cribriform pattern of growth of the tumor and an appropriate immunohistochemical profile consistent with a primary lung adenocarcinoma (CK7/TTF1-positive; CK20/CDX2-negative). For pathologic evaluation, from 5 to 15 slides stained with hematoxylin and eosin were available for review in all cases.

Paraffin blocks for immunohistochemical studies were available in all cases. Four-micrometer sections were stained using a Dako Autostainer Plus according to the manufacturer's protocol. Slides were dried at $60^{\circ} \mathrm{C}$ for $1 \mathrm{~h}$ and deparaffinized. Heat-induced epitope retrieval was performed with Dako Envision FLEX target retrieval solution (high $\mathrm{pH}$ Tris/EDTA) at $100{ }^{\circ} \mathrm{C}$ for $20 \mathrm{~min}$. Primary antibodies (DAKO, Carpinteria, CA, USA) for: CK7 (OVTL 12/30), CK20 (K $\left.\mathrm{K}_{\mathrm{s}} 20.8\right)$, TTF1 (8G7G3/1), thyroglobulin (DAK Tg6), CDX-2 (DAK-CDX2), and ALK (5A4, Novacastra-Leica, Richmond, IL, USA) were incubated at room temperature for $60 \mathrm{~min}$. Signals were detected using a Dako FLEX detection kit. Counterstaining was performed with Envision FLEX hematoxylin for $7 \mathrm{~min}$ at room temperature. Nuclear immunoreactivity was considered positive for TTF1 and CDX-2. Cytoplasmic positivity was considered positive for ALK, thyroglobulin, CK7, and CK20. Appropriate positive and negative controls were run concurrently for all antibodies tested.

\section{DNA Extraction}

Six cases (Medical College of Wisconsin cohort) were amenable to molecular analysis. Hematoxylin and eosin-stained slides were used to identify areas in which tumor cells represented greater than $50 \%$ of total nucleated cells. DNA extraction was performed using Zymo Pinpoint DNA isolation kit (Zymo Research Corporation, Orange, CA, USA) in $10 \mu \mathrm{m}$ unstained sections according to the manufacturer's guidelines. DNA was obtained from both tumor and adjacent, histologically normal appearing areas of each specimen.

\section{EGFR and KRAS Sequencing}

M13-tailed primers were used to amplify exons 18, 19,20 , and 21 of EGFR and a portion of KRAS including codons 12 and 13 (Table 1) by PCR. The PCR amplicons were confirmed by electrophoresis in $2 \%$ agarose gel. PCR products were sequenced in both directions using the BigDye ${ }^{\circledR}$ Direct Cycle Sequencing Kit according to the manufacturer's 
conditions. After the sequencing step, the BigDye Xterminator ${ }^{\circledR}$ Purification Kit was used to clean up the dye excess. Sequence data were obtained with a 3500 genetic analyzer (Applied Biosystems, Foster City, CA, USA). Sequencing results were analyzed using SeqScape v2.7 (Applied Biosystems).

\section{Fluorescence In-Situ Hybridization (FISH)}

FISH was performed on six cases using break apart probes for either ALK, ROS1, or RET (Empire Genomics, Buffalo, NY, USA) according to manufacturer's instructions. Four-micrometer-thick sections of formalin-fixed and paraffin-embedded tumor tissue were deparaffinized, dehydrated, immersed in $0.2 \mathrm{~N} \mathrm{HCl}$, and washed. The sections were immersed in $10 \mathrm{mM}$ citrate buffer and boiled in a microwave for $5 \mathrm{~min}$, treated with pretreatment reagent (Abbott Molecular) followed by protease digestion. After applying the probe, the samples were incubated in a humidified atmosphere using HybriditeTM (Abbott Molecular). Slides were washed with $2 \times$ saline sodium citrate and counterstained with

Table 1 Primers used for EGFR and KRAS mutation analysis

\begin{tabular}{lll}
\hline Gene & Exon & Sequence $\left(5^{\prime} \rightarrow 3^{\prime}\right)$ \\
\hline EGFR & $18 \mathrm{~F}$ & GTGAGGGCTGAGGTGACCCTTGT \\
& $18 \mathrm{R}$ & ATACAGCTTGCAAGGACTCTGGG \\
& $19 \mathrm{~F}$ & AGATCACTGGGCAGCATGTGGCA \\
$19 \mathrm{R}$ & CAGCTGCCAGACATGAGAAAAGG \\
& $20 \mathrm{~F}$ & GAAGCCACACTGACGTGCCTCTCC \\
& $20 \mathrm{R}$ & ACACATATCCCCATGGCAAACTCTT \\
& $21 \mathrm{~F}$ & GCTCAGAGCCTGGCATGAAC \\
& $21 \mathrm{R}$ & AGCATCCTCCCCTGCATGTGT
\end{tabular}

KRAS 1 (Codon 12/13) F AGGTACTGGTGGAGTATTTGATAG 1 (Codon 12/13) R GGTCCTGCACCAGTAATATGCA 4,6-diamino-2-phenylindole II and antifade (p-phenylamide). Signals for each probe were evaluated under a microscope equipped with a double-pass filter (green/orange; Abbott Molecular) and an oil immersion objective lens. Greater than 50 nuclei were analyzed for each case. ALK, ROS1, and RET FISH-positive cases were defined as those presenting more than $15 \%$ split signals ( $>1$ signal diameter separation); in addition, loss of the $5^{\prime}$ ROS 1 probe with retention of the $3^{\prime}$ probe was interpreted as ROS1 FISH positive. ${ }^{25}$

\section{Results}

\section{Clinical Features}

The patients' clinical characteristics are summarized in Table 2. The median age at diagnosis was 64 years (range 30-80 years). Eight patients were men and seven were women. All patients, except one (case 14), were heavy cigarette smokers (greater than 40 cigarettes per day). Co-morbidity was present in two patients; one had sickle cell disease and another had chronic myeloid leukemia. Based on initial histologic examination, the majority of cases were initially interpreted as representing metastases from other organs. Thorough clinical and radiographic examination following the initial diagnosis did not reveal evidence of a primary tumor elsewhere. All patients but one underwent surgical resection as treatment; lobectomy was performed in seven patients, wedge resection in six, one patient had a partial pneumonectomy, and one patient had only a liver biopsy for metastatic disease. Follow-up information was available in six patients; two patients died before 1 year with disseminated disease, four patients were alive without evidence of metastases

Table 2 Clinicopathologic and molecular features in 15 cases of primary cribriform adenocarcinoma of the lung

\begin{tabular}{|c|c|c|c|c|c|c|c|c|}
\hline Case & Sex & $\begin{array}{c}\text { Age } \\
\text { (years) }\end{array}$ & Histologic pattern & Localization & $\begin{array}{c}\text { EGFR } \\
\text { rs105017 }\end{array}$ & KRAS & $\begin{array}{l}\text { Gene } \\
\text { rearrangement }\end{array}$ & Follow-up \\
\hline 1 & $\mathrm{~F}$ & 50 & Prostate/breast-like & RLL & - & - & - & LFU \\
\hline 2 & $\mathrm{M}$ & 50 & Prostate/breast-like & RL & G/A & WT & Negative & LFU \\
\hline 3 & $\mathrm{~F}$ & 76 & Prostate/breast-like & RML & - & - & - & Alive, 10 months \\
\hline 4 & $\mathrm{~F}$ & 71 & Prostate/breast-like & LLL & - & - & - & LFU \\
\hline 5 & $\mathrm{M}$ & 49 & Cystic ovarian/pancreatic adenocarcinoma & RLL & - & - & - & Alive, 4 months \\
\hline 6 & $\mathrm{~F}$ & 55 & Cystic ovarian/pancreatic adenocarcinoma & LUL & - & - & - & Died, 10 months \\
\hline 7 & $\mathrm{~F}$ & 52 & Endometrioid & LLL & G/A & WT & $\operatorname{RET}(17.1 \%)$ & LFU \\
\hline 8 & $\mathrm{~F}$ & 73 & Endometrioid & LUL & G/A & c.G35A & Negative & LFU \\
\hline 9 & M & 80 & Endometrioid & RUL & - & - & - & Alive, 6 months \\
\hline 10 & $\mathrm{M}$ & 68 & Endometrioid & RUL & $\mathrm{A} / \mathrm{A}$ & WT & Negative & LFU \\
\hline 11 & $\mathrm{M}$ & 64 & Intestinal-like & LUL & - & - & - & Died, 6 weeks \\
\hline 12 & $\mathrm{~F}$ & 79 & Intestinal-like & RUL & - & - & - & Alive, 4 months \\
\hline 13 & $\mathrm{M}$ & 57 & Intestinal-like & LUL & - & - & - & LFU \\
\hline 14 & M & 30 & Thyroid-like & RLL & $\mathrm{A} / \mathrm{A}$ & WT & ROS1 (62.5\%) & LFU \\
\hline 15 & $\mathrm{M}$ & 50 & Thyroid-like & RLL & G/A & WT & ALK (46.6\%) & LFU \\
\hline
\end{tabular}

Abbreviations: LFU, lost to follow-up; LUL, left upper lobe; LLL, left lower lobe; RUL, right upper lobe; RML, right middle lobe; RLL, right lower lobe; WT, wild type. rs1050171 is a germline SNP c.2361G/A in exon20 of EGFR. 
at 4 months after surgery. The remainders of patients have been lost to follow-up.

\section{Gross Features}

Fifteen patients had single tumors; tumor size ranged from 1.9 to $7.5 \mathrm{~cm}$. Grossly, the tumors were described as tan-white, well-circumscribed solid masses. Two tumors showed central cavitation. All tumors were peripherally located, with pleural puckering. Six patients had mediastinal lymph node metastases at diagnosis, and one patient had pleural and chest wall metastases.

\section{Histologic Features}

On scanning magnification, all tumors showed a predominant cribriform architecture consisting of glandular spaces lined by cuboidal to cylindrical cells with punched-out round to ovoid lumens. Four cases resembled prostatic or breast adenocarcinomas (Figure 1a). The nests of tumor cells were round to ovoid in shape and separated by abundant desmoplastic stroma. On higher magnification, the cells were cuboidal to polygonal, with centrally placed nuclei containing prominent nucleoli and ample cytoplasm. No goblet cells, signet-ring cells, or other evidence of mucin secretion could be identified. The cribriform spaces resembled 'roman bridges' (Figure 1b). Secretion in the lumens varied from amphophilic to basophilic in two cases, and eosinophilic in two other cases. Areas of comedonecrosis were present in all four cases.

Two cases had abundant mucinous eosinophilic secretion in the glandular lumens. Cribriform structures lined the periphery of cystic spaces in a garland-like pattern, resembling mucinous adenocarcinomas of the pancreas or ovary (Figure 2a). The tumor nests were separated by scant fibrous stroma. Cells varied from cuboidal to cylindrical, their cytoplasm was eosinophilic and the nuclei were oval or elongated with some pseudostratification. Atypia was moderate to severe and there were frequent mitoses (Figure 2b).

Four cases resembled adenocarcinoma of the endometrium, with tumor islands containing cribriform structures separated by desmoplastic stroma (Figure 3a). The cells were cylindrical, with ovoid nuclei containing two or three small nucleoli and well-defined cell borders. All cases showed, at least focally, subnuclear vacuoles resembling periovulatory-phase endometrium, and dirty necrosis in the lumen (Figure 3b). One of these cases also showed areas that resembled ovarian mucinous cystadenocarcinoma, with cystic spaces rich in mucin, cribriform architecture, and more solid foci with structures resembling morules; however, the solid areas were separate from the cribriform areas and did not show squamoid features as are seen in true morules.

Three cases showed features that simulated intestinal-type colorectal adenocarcinoma (Figure 4a), with extensive areas of necrosis, cystically dilated tumor islands with cribriform structures adopting a garland-like arrangement at their periphery, and adjacent areas with small confluent cribriform nests with necrosis in their lumens. Tumor cells were columnar and showed marked nuclear atypia, with elongated nuclei, increased nuclear chromatin, and ample eosinophilic cytoplasm. Scattered goblet cells were present. Thick mucoid eosinophilic secretions were present in the lumens admixed with inflammatory cells and nuclear debris (ie, 'dirty' necrosis; Figure 4b).

Two cases simulated metastases from thyroid papillary carcinoma and showed a striking cribriform pattern admixed with small papillary tufts (Figure 5a). The tumor cells were round or oval and
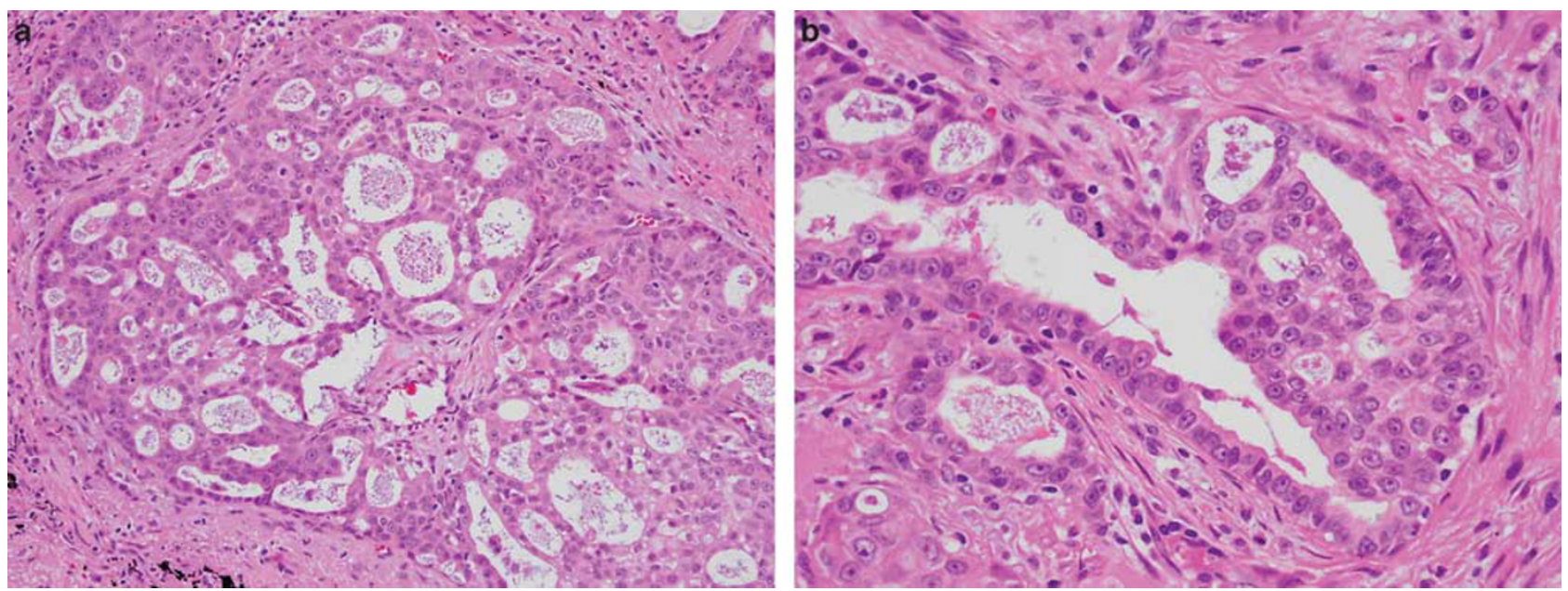

Figure 1 (a) Cribriform adenocarcinoma of lung showing tumor cell islands with punched-out cribriform spaces lined by cuboidal to polygonal epithelial cells. The structures are reminiscent of cribriform ductal carcinoma of the breast (case 1). (b) Higher magnification from the same case shows punched-out lumens with rigid columns of cells resembling roman bridges. 

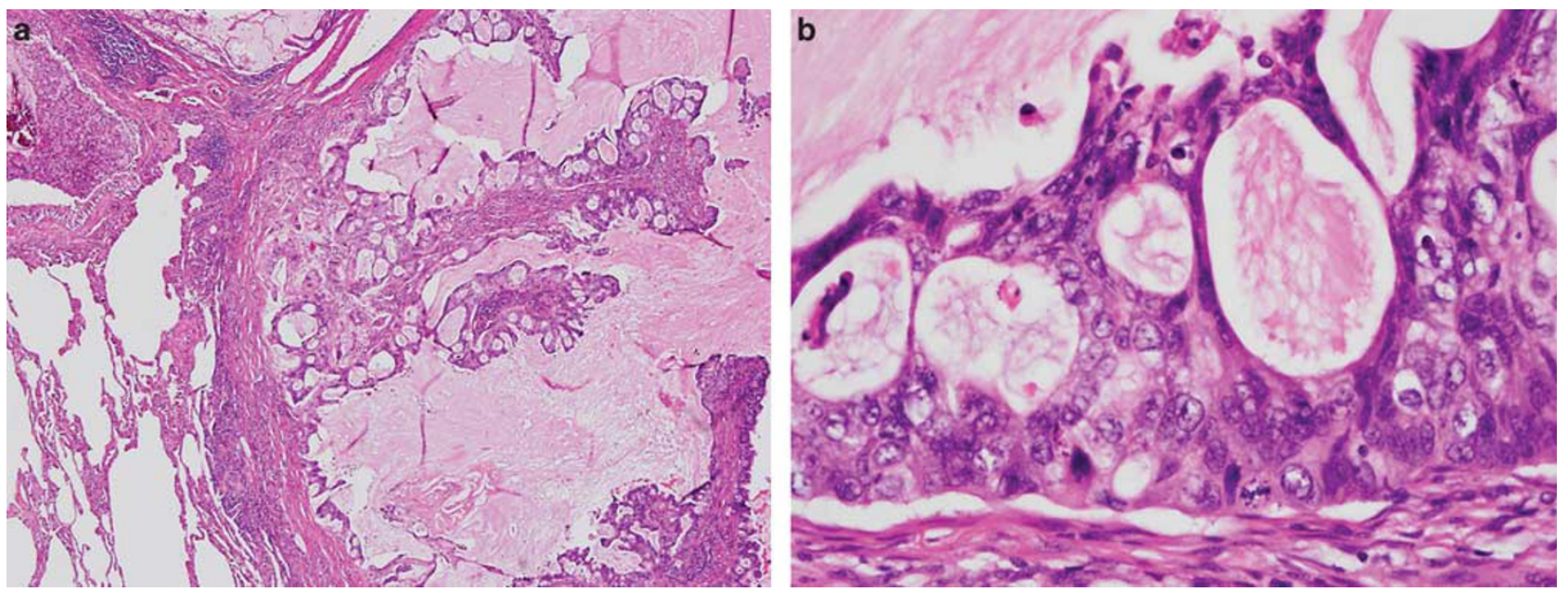

Figure 2 (a) Lung mucinous adenocarcinoma with prominent cribriform spaces are seen arranged in a garland-like distribution at the periphery resembling metastasis from mucinous cystadenocarcinoma of pancreas or ovary (case 5). (b) Higher magnification shows punched-out luminal spaces surrounded by cells with marked nuclear atypia and mitotic figures.
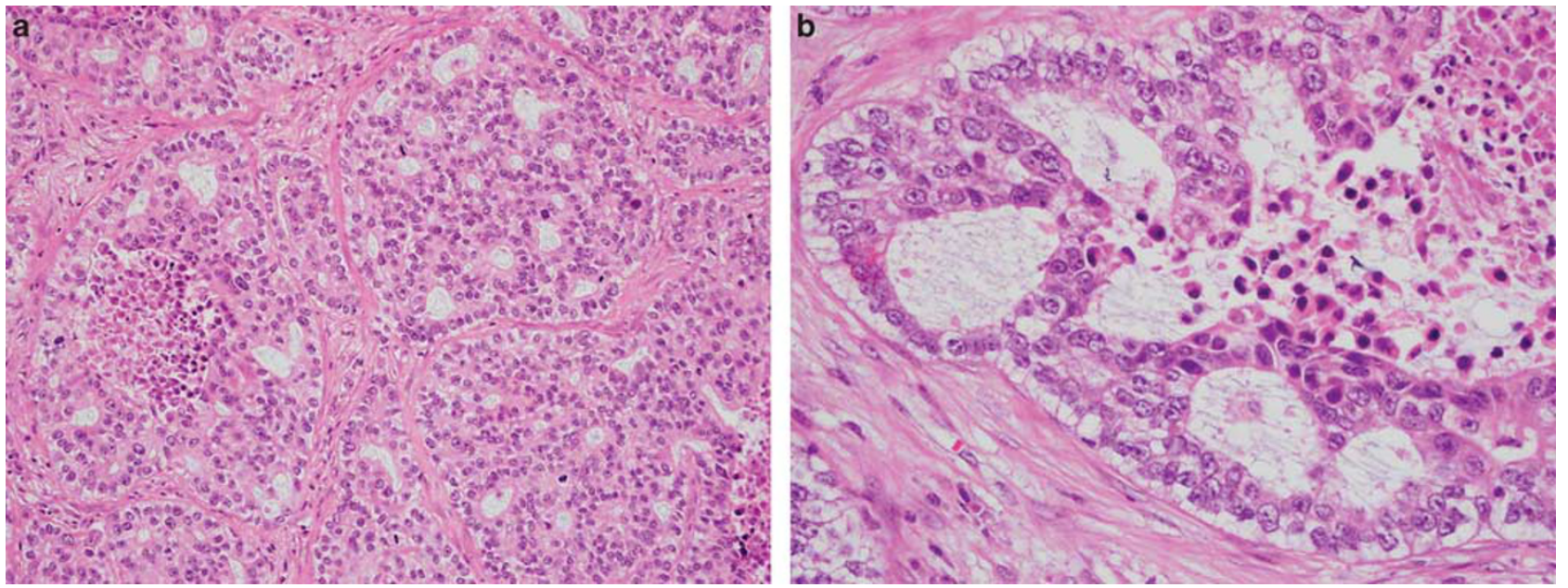

Figure 3 (a) Cribriform adenocarcinoma of lung showing multiple punched-out cribriform spaces and foci of comedonecrosis resembling endometrioid adenocarcinoma (case 7). (b) Higher magnification of area showing prominent subnuclear vacuoles resembling periovulatory endometrium.
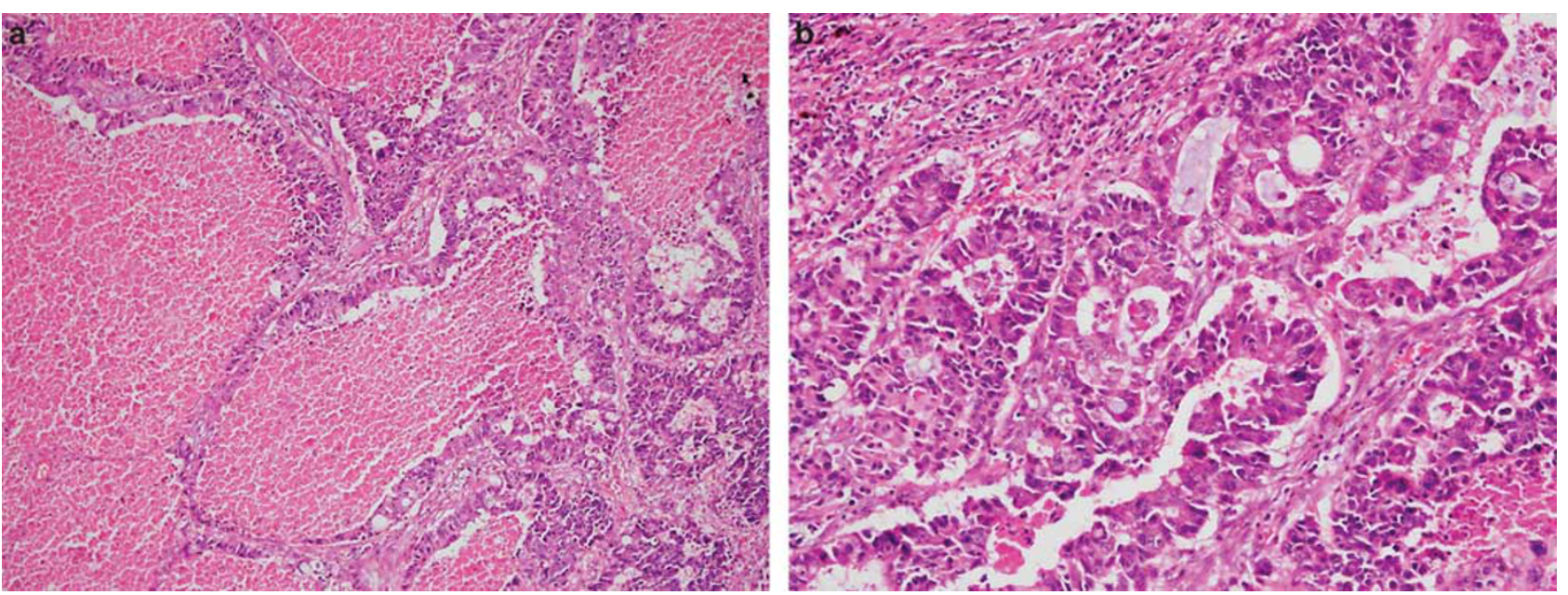

Figure 4 (a) Intestinal-type adenocarcinoma of lung showing dilated glandular spaces filled with hemorrhage and necrotic debris and showing focal cribriform growth pattern (case 12). (b) Higher magnification shows cribriform glandular structures lined by tall columnar cells admixed with a few goblet cells. 
showed marked clearing of the nuclear chromatin, scattered longitudinal nuclear grooves, and rare intranuclear eosinophilic inclusions as well as overlapping of nuclei (Figure 5b). The lumens in the cribriform areas were filled with an eosinophilic secretion with scalloping and occasional macrophages. Scattered psammoma bodies were also present. Both cases also showed focal areas displaying a lepidic, bronchioloalveolar-type pattern of growth in adjacent alveoli.

\section{Immunohistochemistry Analysis}

All cases were positive for TTF-1 and CK7 (Figures $6 \mathrm{a}$ and $\mathrm{b}$ ). None of the cases showed positivity for CK20 or CDX-2. Case 15 showed positive staining of the tumor cells for ALK antibody (Figure 7). Thyroglobulin was negative in both cases that showed a thyroid-like pattern (cases 14 and 15).

\section{Molecular Studies}

No activating EGFR mutations were identified. EGFR exon 20 contains a synonymous singlenucleotide polymorphism (refSNP ID rs1050171) p.Q787Q (c.2361G > A), ${ }^{26}$ with the ancestral allele of $\mathrm{G}$. The minor allele frequency of $\mathrm{A}$ is 0.418 . Four patients were heterozygous G/A (cases 2, 7, 8, 15) and two patients were homozygous A/A (cases 10 and 14) for this SNP. For each case, sequence of paired normal and tumor DNA demonstrated the same genotype indicating this is a germline singlenucleotide polymorphism that does not correlate with either histology or activating EGFR mutation. Dideoxy sequencing demonstrated the activating KRAS mutation c.G35A (p.G12D) in a tumor with endometrioid-like cribriform histology (case 8). Both cases with thyroid-like histology demonstrated gene rearrangements by FISH (Figure 7) with one case harboring a ROS1 rearrangement (case 14) and the other an $A L K$ rearrangement (case 15). Case 7 with endometrioid histology demonstrated a RET gene rearrangement in a small number of malignant nuclei $(17.1 \%)$.

\section{Discussion}

Lung adenocarcinomas are characterized by histologic heterogeneity; adenocarcinomas with a single pure or predominant histologic pattern are rare. ${ }^{27}$ Cribriform architecture is not a characteristic pattern in lung adenocarcinoma and tumors displaying this unusual growth pattern are not included in the World Health Organization classification of lung tumors. ${ }^{3}$ In the literature, cribriform adenocarcinomas of the lung, when mentioned, are grouped with the acinar subtype $e^{5,28}$ and, according to the actual recommendations by the International Association for the Study of Lung Cancer/American Thoracic Society/European Respiratory Society, there are not sufficient data to separate them from other subtypes. ${ }^{7}$ Because of their rarity, the presence of a predominant or exclusive cribriform growth pattern in a lung tumor of an adult or elderly patient generally raises the diagnostic possibility of a metastasis from another organ.

We have described 15 primary lung adenocarcinomas with a predominant cribriform architecture. The majority of the lesions were initially mistaken for examples of metastases to the lung from carcinomas from various other organs. Immunohistochemical studies coupled with thorough clinical investigation supported the primary lung origin of these tumors.

Primary lung tumors with a cribriform architecture resembling colorectal carcinomas were first
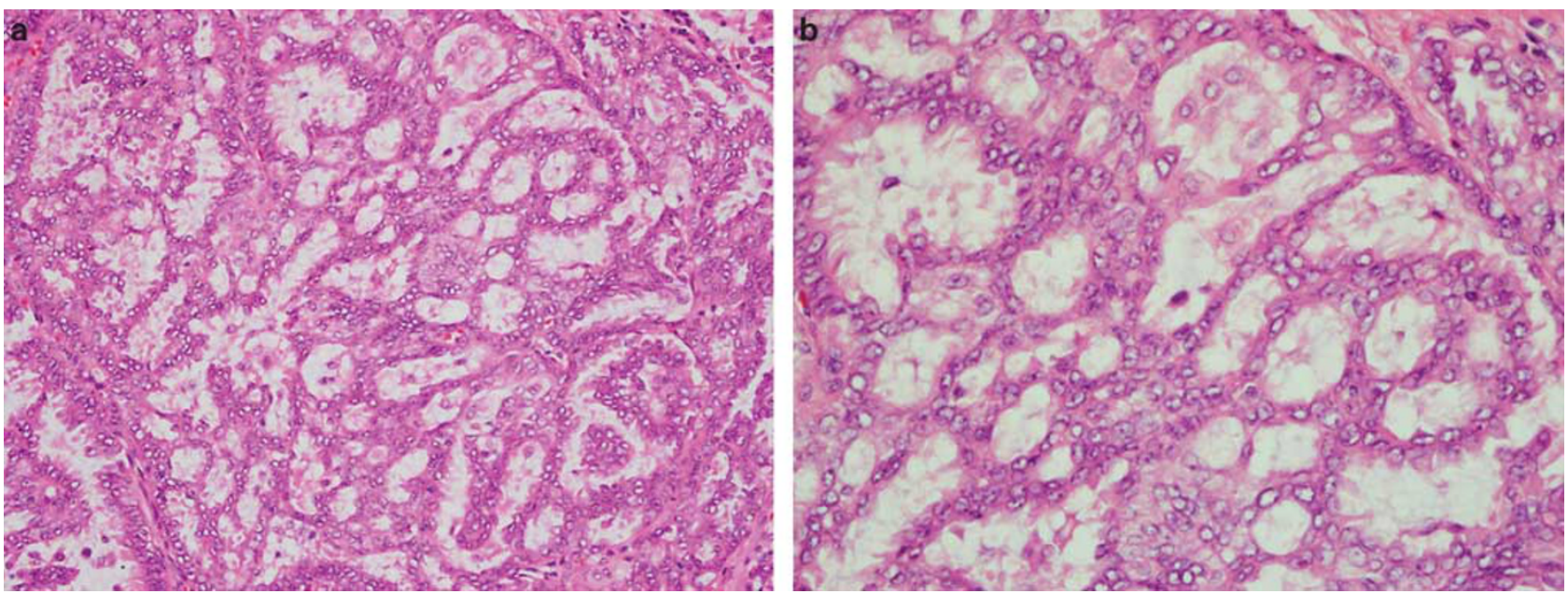

Figure 5 (a) Cribriform adenocarcinoma of lung with focal papillary areas (lower right) showing clearing of the nuclear chromatin in the tumor cells simulating a metastasis of papillary thyroid carcinoma (case 14). (b) Higher magnification showing prominent clearing of the nuclear chromatin and overlapping of nuclei. 

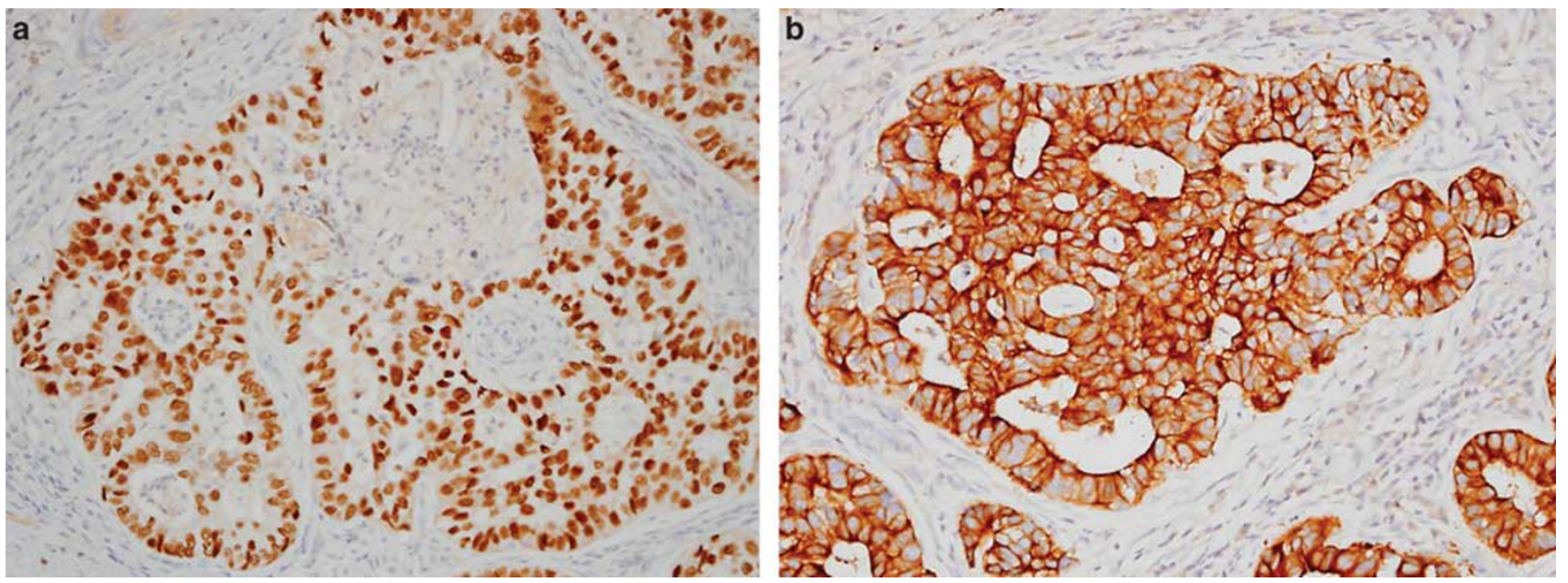

Figure 6 (a) Cribriform adenocarcinoma of lung showing strong nuclear immunoreactivity for TTF-1 (case 1). (b) Cribriform adenocarcinoma of lung showing cytoplasmic immunoreactivity for CK7 (case 1).

described by Tsao and Fraser. ${ }^{29}$ The tumors were characterized by a predominant component of neoplastic tall columnar cells admixed with goblet cells and Paneth cells, with occasional features of neuroendocrine differentiation. More recently, pulmonary intestinal-type adenocarcinomas have also been described by Yousem ${ }^{30}$ and by Inamura. ${ }^{31}$ The tumors described by Yousem were characterized by a garland-like architecture, central 'dirty' necrosis, and stratified tall columnar cells lacking significant goblet cell or signet-ring features lining the glands. The author remarked that the tumors closely resembled colorectal and sinonasal 'intestinaltype' adenocarcinomas. A cribriform arrangement of the neoplastic cells in which luminal spaces were filled with mucinous material and necrotic debris was a feature of those tumors. Based on the patterns of immunoreactivity, pulmonary adenocarcinomas with enteric features can be divided into those showing positivity for TTF-1/CK7 and negativity for CDX2/CK20 (ie, lung adenocarcinoma with enteric morphology), ${ }^{30}$ and those showing CD20/CDX2 positivity and negative CK7/TTF1 (ie, lung adenocarcinoma with enteric differentiation). ${ }^{31,32}$ The majority of the enteric-type tumors, however, appear to exhibit a primary lung phenotype and, thus, display only enteric morphology.

Some of the cases in our study showed some of the features described by Yousem ${ }^{30}$ and Inamura ${ }^{31}$ resembling colorectal carcinoma, but our series further expands the morphologic spectrum that these tumors can display. This is of importance for differential diagnosis as tumors other than colorectal adenocarcinomas demonstrating a prominent cribriform growth pattern are capable of metastasizing to the lung and can arise from diverse sites such as the gallbladder, ${ }^{33}$ paranasal sinuses, ${ }^{34}$ urinary bladder, ${ }^{35}$ breast, ${ }^{11}$ prostate, ${ }^{10}$ pancreas, ${ }^{36}$ salivary glands, ${ }^{37}$ thyroid, ${ }^{38}$ sweat glands, stomach, ovary, ${ }^{39}$ uterus, and endocervix. ${ }^{40}$
Adenocarcinomas of the prostate and breast can metastasize to the lung and can often display a cribriform architecture with comedonecrosis. ${ }^{3}$ In a study of 129 frozen sections from 121 patients with breast adenocarcinoma and lung nodules, Herbst et $a l^{11}$ found that comedonecrosis within solid tumor nests and cribriform architecture favored a diagnosis of breast metastases. A study of metastatic adenocarcinomas from the prostate to the lung also showed that the most common features in such tumors were a cribriform growth pattern with comedonecrosis, blue mucin, and mild pleomorphism. ${ }^{10}$ All of our cases with a prostate/breast cribriform pattern except for one were women, TTF-1 was diffusely positive, and none had a previous history or evidence of breast or prostate carcinoma.

Cystadenocarcinomas from the pancreatobiliary tract and the ovary can metastasize to the lung and show a cribriform pattern. Endometrioid adenocarcinomas from the uterus typically have a back to back cribriform arrangement, with cylindrical cells and occasional dirty necrosis in their lumens., ${ }^{7,36}$ Application of immunohistochemical stains can be of help in this setting; although such tumors can show reactivity for CK7, pancreatic, endometrioid, and ovarian carcinomas are generally negative for TTF1.

Papillary carcinomas of the thyroid frequently metastasize to regional lymph nodes and the lung is the principal distant site affected. Distant metastases very rarely can be the first manifestation of the disease. ${ }^{38}$ The cribriform-morular variant of papillary thyroid carcinoma is another tumor that can metastasize to the lung late in its course. This tumor is characterized by prominent cribriform architecture in association with squamoid morules. The tumor cells are cylindrical with vacuolated nuclei, nuclear grooves, prominent nucleoli, and occasional pseudoinclusions. Immunohistochemical stains are positive for TTF-1, CK7, and nuclear $\beta$-catenin. 

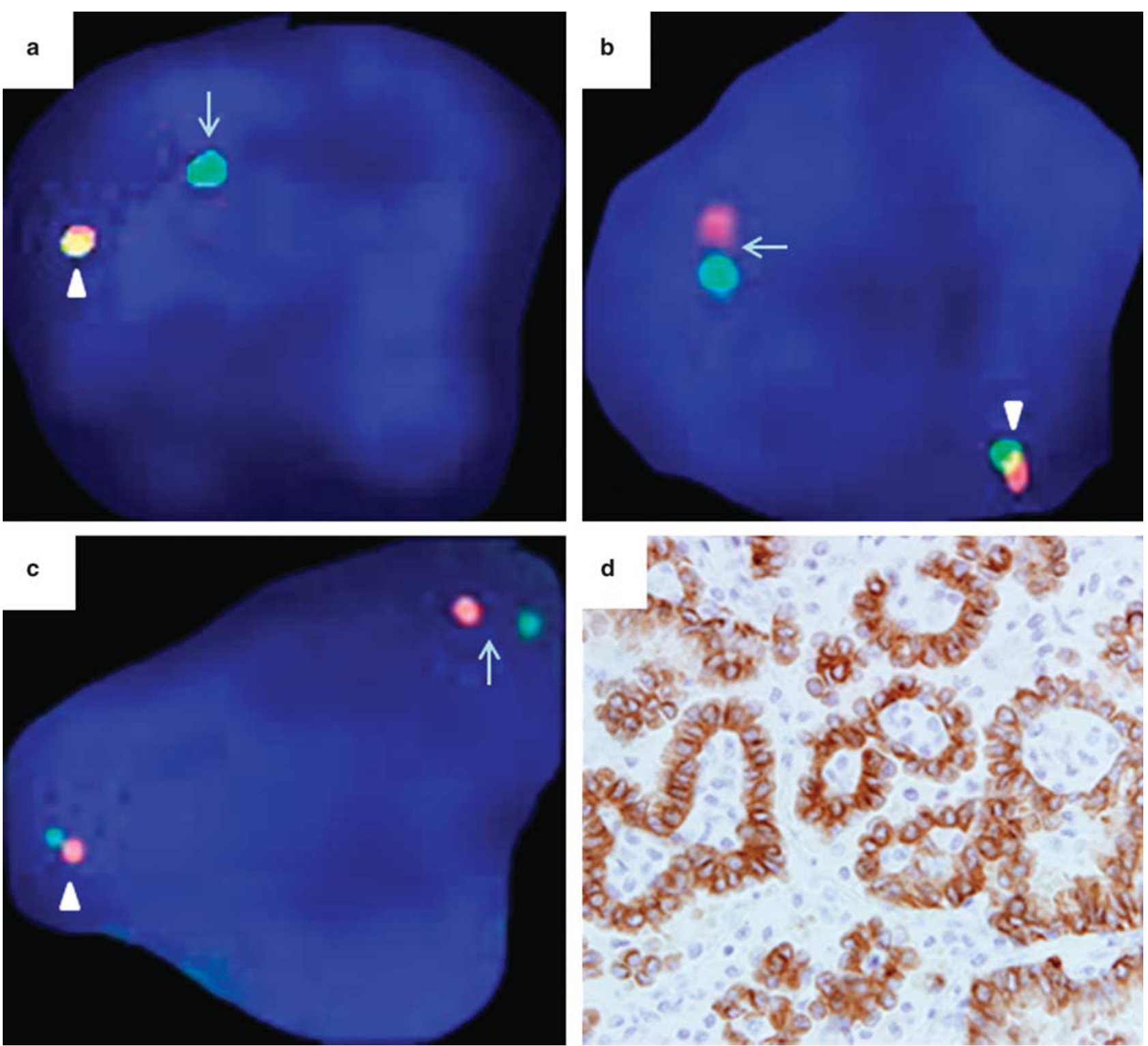

Figure 7 (a) ROS1 translocation indicated by loss of $5^{\prime}$ (red) signal with retention of $3^{\prime}$ (green) signal in $62.5 \%$ of nuclei. (b) RET $5^{\prime}$ and $3^{\prime}$ probes split in $17.1 \%$ of nuclei. (c) EML4-ALK rearrangement in $46.6 \%$ of nuclei. Overlapping probes (arrowhead) indicate intact chromosomes. Split probes (arrows) indicate chromosomes in which the target gene is disrupted due to chromosomal translocation. (d) Same case as $\mathbf{c}$ demonstrating ALK expression by IHC.

Up to $40 \%$ may show neuroendocrine differentiation. ${ }^{34}$ Our thyroid-like cases shared the nuclear features of papillary thyroid carcinoma as well as having areas with a cribriform pattern; however, they lacked squamoid morules. Moreover, thyroglobulin stains performed in both cases were negative and physical and ultrasound examination of the head and neck did not reveal evidence of thyroid nodules or masses.

Finally, a more remote possibility in the differential diagnosis is the so-called 'fetal type' adenocarcinoma of the lung, a rare variant of primary lung adenocarcinoma seen mostly in young adults. Histologically, it is characterized by a prominent cribriform architecture with abundant clear glycogen-rich cytoplasm, subnuclear vacuoles, and the distinctive presence of squamoid morules located predominantly at the base of the glands. These tumors also stain strongly for TTF1 and may display aberrant nuclear positivity for $\beta$-catenin. ${ }^{7,41}$ Four of our cases contained areas with clear cells; however, none of our cases showed the distinctive combination of back-to-back arrangement of glandular spaces, subnuclear vacuoles, and squamoid morules. Only one of our cases showed focally clear subnuclear vacuoles as well as non-squamoid solid areas resembling morules; however, the glands were not back to back, and the morule-like structures 
were not associated with the cribriform glands but were located within dilated glandular spaces at the periphery of the tumor.

The $A L K, R O S$, and RET gene rearrangements leading to constitutive kinase activity define a new molecular subtype of lung adenocarcinoma, and patients with $A L K$ or $R O S 1$ gene rearrangements demonstrate benefit from criztotinib. Two of our cases showed a gene rearrangement involving EML4$A L K$ or ROS1, both of which had thyroid-like morphology that simulated metastatic papillary thyroid carcinoma. Although previous reports have demonstrated that $A L K$ and ROS1 gene rearrangements frequently occur in young, non-smoking patients with predominantly cribriform, solid, or signet-ring cell patterns, these features have no specific role in selecting patients for molecular testing; rather molecular testing is recommended in lung cancer patients with adenocarcinoma and mixed lung cancer with an adenocarcinoma component. ${ }^{4,14,23,25,42-47}$ The KRAS codon 12 c.G35A (p.G12D) mutation was present in one case with a pure cribriform architecture. No activating EGFR mutations were identified in our cases.

In summary, we have described a group of primary lung adenocarcinomas that are characterized by a distinctive and predominantly cribriform histology. All except one occurred in older patients with a heavy smoking history and were initially suspected of representing metastases from various other organs. Careful clinicopathologic assessment and immunohistochemical studies helped establish the primary pulmonary origin of these tumors. Last, an activating KRAS mutation and alterations in both $A L K$ and ROS1 were observed in a subset of the cases amenable to analysis. The spectrum of activating mutations and genetic variation observed in this series is similar in frequency and distribution to conventional lung adenocarcinoma and do not seem to identify a particular molecular signature or predictive therapeutic response in lung adenocarcinoma with cribriform histology. However, because of their distinctive morphology, awareness of this unusual histologic variant of lung adenocarcinoma is of importance to avoid misdiagnosis with a metastasis from an occult or distant primary.

\section{Acknowledgments}

We acknowledge the assistance of Xiuxu Chen and Kathryn Stoll with the preparation of this manuscript. This study was funded by the Clinical and Translational Research Lab, Department of Pathology, Medical College of Wisconsin, Milwaukee, Wisconsin.

\section{Disclosure/conflict of interest}

The authors declare no conflict of interest.

\section{References}

1 Cetin K, Ettinger DS, Hei YJ, et al. Survival by histologic subtype in stage IV nonsmall cell lung cancer based on data from the Surveillance, Epidemiology and End Results Program. Clin Epidemiol 2011;3:139-148.

2 Chilosi M, Murer B. Mixed adenocarcinomas of the lung: place in new proposals in classification, mandatory for target therapy. Arch Pathol Lab Med 2010;134: 55-65.

3 Travis WD, Brambilla E, Muller-Hermelink HK, et al. Pathology and Genetics of Tumours of the Lung, Pleura, Thymus, and Heart. IARC Press: France: Lyon; 2004, pp 344.

4 Cooper WA, O'Toole S, Boyer M, et al. What's new in non-small cell lung cancer for pathologists: the importance of accurate subtyping, EGFR mutations and ALK rearrangements. Pathology 2011;43:103-115.

5 Kerr KM. Pulmonary adenocarcinomas: classification and reporting. Histopathology 2009;54:12-27.

6 Motoi N, Szoke J, Riely GJ, et al. Lung adenocarcinoma: modification of the 2004 WHO mixed subtype to include the major histologic subtype suggests correlations between papillary and micropapillary adenocarcinoma subtypes, EGFR mutations and gene expression analysis. Am J Surg Pathol 2008;32:810-827.

7 Travis WD, Brambilla E, Noguchi M, et al. International association for the study of lung cancer/american thoracic society/european respiratory society international multidisciplinary classification of lung adenocarcinoma. J Thorac Oncol 2011;6:244-285.

8 Russell PA, Wainer Z, Wright GM, et al. Does lung adenocarcinoma subtype predict patient survival?: A clinicopathologic study based on the new International Association for the Study of Lung Cancer/American Thoracic Society/European Respiratory Society international multidisciplinary lung adenocarcinoma classification. J Thorac Oncol 2011;6:1496-1504.

9 Yoshizawa A, Motoi N, Riely GJ, et al. Impact of proposed IASLC/ATS/ERS classification of lung adenocarcinoma: prognostic subgroups and implications for further revision of staging based on analysis of 514 stage I cases. Mod Pathol 2011;24:653-664.

10 Copeland JN, Amin MB, Humphrey PA, et al. The morphologic spectrum of metastatic prostatic adenocarcinoma to the lung: special emphasis on histologic features overlapping with other pulmonary neoplasms. Am J Clin Pathol 2002;117:552-557.

11 Herbst J, Jenders R, McKenna R, et al. Evidence-based criteria to help distinguish metastatic breast cancer from primary lung adenocarcinoma on thoracic frozen section. Am J Clin Pathol 2009;131:122-128.

12 Takeuchi K, Soda M, Togashi Y, et al. RET, ROS1 and ALK fusions in lung cancer. Nat Med 2012;18:378-381.

13 Keedy VL, Temin S, Somerfield MR, et al. American Society of Clinical Oncology provisional clinical opinion: epidermal growth factor receptor (EGFR) Mutation testing for patients with advanced nonsmall-cell lung cancer considering first-line EGFR tyrosine kinase inhibitor therapy. J Clin Oncol 2011;29: 2121-2127.

14 Mino-Kenudson M, Mark EJ. Reflex testing for epidermal growth factor receptor mutation and anaplastic lymphoma kinase fluorescence in situ hybridization in non-small cell lung cancer. Arch Pathol Lab Med 2011;135:655-664. 
15 Pirker R, Herth FJ, Kerr KM, et al. Consensus for EGFR mutation testing in non-small cell lung cancer: results from a European workshop. J Thorac Oncol 2010;5: 1706-1713.

16 Lindeman NI, Cagle PT, Beasley MB, et al. Molecular testing guideline for selection of lung cancer patients for EGFR and ALK tyrosine kinase inhibitors: guideline from the College of American Pathologists, International Association for the Study of Lung Cancer, and Association for Molecular Pathology. Arch Pathol Lab Med 2013;137:828-860.

17 Rikova K, Guo A, Zeng Q, et al. Global survey of phosphotyrosine signaling identifies oncogenic kinases in lung cancer. Cell 2007;131:1190-1203.

$18 \mathrm{Ju}$ YS, Lee WC, Shin JY, et al. A transforming KIF5B and RET gene fusion in lung adenocarcinoma revealed from whole-genome and transcriptome sequencing. Genome Res 2012;22:436-445.

19 Kohno T, Ichikawa H, Totoki Y, et al. KIF5B-RET fusions in lung adenocarcinoma. Nat Med 2012;18:375-377.

20 Lipson D, Capelletti M, Yelensky R, et al. Identification of new ALK and RET gene fusions from colorectal and lung cancer biopsies. Nat Med 2012;18:382-384.

21 Oxnard GR, Binder A, Janne PA. New targetable oncogenes in non-small-cell lung cancer. J Clinical Oncol 2013;31:1097-1104.

22 Drilon A, Wang L, Hasanovic A, et al. Response to Cabozantinib in patients with RET fusion-positive lung adenocarcinomas. Cancer Discov 2013;3:630-635.

23 Lindeman NI, Cagle PT, Beasley MB, et al. Molecular testing guideline for selection of lung cancer patients for EGFR and ALK tyrosine kinase inhibitors: guideline from the College of American Pathologists, International Association for the Study of Lung Cancer, and Association for Molecular Pathology. J Mol Diag 2013;15:415-453.

24 Kakegawa S, Shimizu K, Sugano M, et al. Clinicopathological features of lung adenocarcinoma with KRAS mutations. Cancer 2011;117:4257-4266.

25 Yoshida A, Kohno T, Tsuta K, et al. ROS1-rearranged lung cancer: a clinicopathologic and molecular study of 15 surgical cases. Am J Surg Pathol 2013;37:554-562.

26 Yeh YC, Chou TY. Pulmonary adenocarcinoma with microcystic histology and intratumoral heterogeneity of EGFR gene polymorphism. Histopathology 2010;57: 112-120.

27 Sica G, Yoshizawa A, Sima CS, et al. A grading system of lung adenocarcinomas based on histologic pattern is predictive of disease recurrence in stage I tumors. Am J Surg Pathol 2010;34:1155-1162.

28 Shaw AT, Solomon B. Targeting anaplastic lymphoma kinase in lung cancer. Clin Cancer Res 2011;17: 2081-2086.

29 Tsao MS, Fraser RS. Primary pulmonary adenocarcinoma with enteric differentiation. Cancer 1991;68: 1754-1757.

30 Yousem SA. Pulmonary intestinal-type adenocarcinoma does not show enteric differentiation by immunohistochemical study. Mod Pathol 2005;18:816-821.

31 Inamura K, Satoh Y, Okumura S, et al. Pulmonary adenocarcinomas with enteric differentiation: histologic and immunohistochemical characteristics compared with metastatic colorectal cancers and usual pulmonary adenocarcinomas. Am J Surg Pathol 2005; 29:660-665.

32 Canney A, Sheahan K, Keegan D, et al. Synchronous lung tumours in a patient with metachronous color- ectal carcinoma and a germline MSH2 mutation. J Clin Pathol 2009;62:471-473.

33 Albores-Saavedra J, Henson DE, Moran-Portela D, et al. Cribriform carcinoma of the gallbladder: a clinicopathologic study of 7 cases. Am J Surg Pathol 2008;32: 1694-1698.

34 Cameselle-Teijeiro J, Menasce LP, Yap BK, et al. Cribriform-morular variant of papillary thyroid carcinoma: molecular characterization of a case with neuroendocrine differentiation and aggressive behavior. Am J Clin Pathol 2009;131:134-142.

35 Tamboli P, Mohsin SK, Hailemariam S, et al. Colonic adenocarcinoma metastatic to the urinary tract versus primary tumors of the urinary tract with glandular differentiation: a report of 7 cases and investigation using a limited immunohistochemical panel. Arch Pathol Lab Med 2002;126:1057-1063.

36 Meriden Z, Yemelyanova AV, Vang R, et al. Ovarian metastases of pancreaticobiliary tract adenocarcinomas: analysis of 35 cases, with emphasis on the ability of metastases to simulate primary ovarian mucinous tumors. Am J Surg Pathol 2011;35:276-288.

37 Skalova A, Sima R, Kaspirkova-Nemcova J, et al. Cribriform adenocarcinoma of minor salivary gland origin principally affecting the tongue: characterization of new entity. Am J Surg Pathol 2011;35:1168-1176.

38 Angeles-Angeles A, Chable-Montero F, Martinez-Benitez B, et al. Unusual metastases of papillary thyroid carcinoma: report of 2 cases. Ann Diag Pathol 2009;13:189-196.

39 DeCostanzo DC, Elias JM, Chumas JC. Necrosis in 84 ovarian carcinomas: a morphologic study of primary versus metastatic colonic carcinoma with a selective immunohistochemical analysis of cytokeratin subtypes and carcinoembryonic antigen. Int J Gynecol Pathol 1997;16:245-249.

40 Toker C. Tumors: An Atlas of Differential Diagnosis, 1st edn Lippincott Williams and Wilkins 1983, pp 216-217.

41 Nakatani Y, Masudo K, Miyagi Y, et al. Aberrant nuclear localization and gene mutation of beta-catenin in low-grade adenocarcinoma of fetal lung type: upregulation of the Wnt signaling pathway may be a common denominator for the development of tumors that form morules. Mod Pathol 2002;15:617-624.

42 Inamura K, Takeuchi K, Togashi Y, et al. EML4-ALK lung cancers are characterized by rare other mutations, a TTF-1 cell lineage, an acinar histology, and young onset. Mod Pathol 2009;22:508-515.

43 Inamura K, Takeuchi K, Togashi Y, et al. EML4-ALK fusion is linked to histological characteristics in a subset of lung cancers. J Thorac Oncol 2008;3:13-17.

44 Rodig SJ, Mino-Kenudson M, Dacic S, et al. Unique clinicopathologic features characterize ALK-rearranged lung adenocarcinoma in the western population. Clin Cancer Res 2009;15:5216-5223.

45 Takahashi T, Sonobe M, Kobayashi M, et al. Clinicopathologic features of non-small-cell lung cancer with EML4-ALK fusion gene. Ann Surg Oncol 2010;17:889-897.

46 Yoshida A, Tsuta K, Nakamura H, et al. Comprehensive histologic analysis of ALK-rearranged lung carcinomas. Am J Surg Pathol 2011;35:1226-1234.

47 Wong DW, Leung EL, So KK, et al. The EML4-ALK fusion gene is involved in various histologic types of lung cancers from nonsmokers with wild-type EGFR and KRAS. Cancer 2009;115:1723-1733. 